\title{
Effect of Electron Beam and Gamma Rays on Carbon Nanotube Yarn Structure
}

\author{
Maria Cecilia Evora ${ }^{a, b} *$, Nitilaksha Hiremath ${ }^{b}$,Xinyi Lu ${ }^{c}$, Nam-Goo Kang ${ }^{c}$, Leonardo Gondin de \\ Andrada e Silva ${ }^{\text {, Gajanan Bhat }}$, Jimmy Mays ${ }^{c}$ \\ anstituto de Estudos Avançados - IEAV/DCTA, São José dos Campos, SP, Brazil \\ ${ }^{b}$ Department of Materials Science and Engineering, University of Tennessee, Knoxville, TN, USA \\ ${ }^{c}$ Department of Chemistry, University of Tennessee, Knoxville, TN, USA \\ ${ }^{d}$ Instituto de Pesquisas Energéticas e Nucleares - IPEN, Av. Prof lineu Prestes, 2242, Cidade \\ Universitária, 05508-000, SP, Brazil
}

Received: January 17, 2017; Revised: July 06, 2017; Accepted: August 17, 2017

\begin{abstract}
Individual carbon nanotubes (CNTs) exhibit excellent mechanical, electrical and thermal properties, leading to development of a new generation of advanced lightweight materials and spacecraft electronics substituting the electronics based on silicon. The direct assembly of CNTs into macroscopic fibers or sheets has been a way to overcome their dispersion and processing challenges. Because of a wide range of applications of this material, we investigate effectively the defects on CNT yarns structures created by electron beam and gamma sources and their impact on the morphology and mechanical properties. The irradiated samples with electron beam at doses of 400,600 and $800 \mathrm{kGy}$ had a decrease in the strength from $219.60 \pm 18.90 \mathrm{MPa}$ for pristine yarn to $108.86 \pm 23.77,153.15 \pm 21.63,170.50 \pm$ $25.78 \mathrm{MPa}$, respectively. The sample irradiated with gamma in air at dose of $100 \mathrm{kGy}$ had the strength increased slightly as compared with the pristine sample and an increase in the elasticity modulus from $8.79 \pm 1.19$ to $19.63 \pm 2.02 \mathrm{GPa}$ as compared to CNT pristine yarn. The quality of the CNT yarns that was gamma irradiated in air with absorbed dose of $100 \mathrm{kGy}$ was not affected by the radiation process with improvement of $123 \%$ of the Young's modulus.
\end{abstract}

Keywords: Carbon nanotube yarn, electron beam, gamma rays.

\section{Introduction}

CNT yarns were developed to overcome the CNT dispersion issue and, despite the production challenges and expenses, it is attractive material due their high strength, stiffness, thermal and electrical conductivity. It is made of aligned multiwall carbon nanotubes (MWCNTs) that are held together through van der Waals interactions and entanglements that can translate the nanoscale properties of CNTs to the micro and mesoscale ${ }^{1}$.

There are two main methods to produce CNTs: liquid and solid state spinning processes. The principal issue in the liquid state process is determined by the original difficulty to disperse CNTs in organic and aqueous solvents, hard to align in solution and CNTs do not melt due to high stiffness and molecular weight ${ }^{2}$. In the solid state spinning process, the fiber is spun directly from CVD reactor (aerogel method) and, also, the CNT yarn can be spun from CNT grown on a substrate. In this paper, we investigated the CNT yarn produced via solid state spinning. It is a process that leads to better mechanical properties but the continuous spinning makes the shear interaction and structural organization of the internal CNTs become problematic. It is a wonderful material to overcome single CNT limitations but, because of defect that may be created during fabrication process and only the

*e-mail: evoramac@hotmail.com
Van de Walls force that hold them together, it is not enough to efficiently transfer load between CNTs, it is necessary to improve the shear interactions ${ }^{3-5}$. Just for comparison PANbased carbon fibers produced by Toray (Japan) has 4.6 and $230 \mathrm{GPa}$ for tensile strength and modulus, respectively ${ }^{6}$.

Carbon nanostructures are very sensitive to radiation and this sensitivity can be exploited for controllable modification of their structural properties such as converting $\mathrm{sp}^{2}$ hybridized carbon into $\mathrm{sp}^{3}$ or forming entirely new structures. Cross linking is still the subject of investigations to understand the nature of this process because tubes inside the ropes are separately by $0.34 \mathrm{~nm}$ that it is 2 times the van der Waals radius of the carbon atom. This large distance makes it difficult to bridge carbons by single $\mathrm{sp}^{3}$ bonds ${ }^{7}$. Radiation cross linking is an alternative and environmentally friendly way to create modification within the fiber structure. High energy electrons, primary or secondary electrons, can generate atomic scale defects due to transfer of energy (kinetic energy). This energy should be enough to dislocate the target atom from the original position in the lattice and promote points of defects that may generate cross-linking process or large dimension defects (clustering of vacancies). E-beam energy (called energy threshold) greater than 80 kev is able to displace carbon atoms from hexagonal lattice network of CNT. It is important to point out that this energy threshold will depend on the local arrangement of carbon 
atoms relative to the electron beam and the type of $\mathrm{CNT}^{8}$. With the dislocation of a carbon atom, the quick process of recombination takes place and, consequently, points of defects are created that can bridge adjacent layers and promote crosslinking due to di-vacancy, interstitial and the nearest neighbor Frankel pair of defects formation or /and larger defects are created in plane which is responsible for the strength drastically reduced ${ }^{9}$.

The study of effect of radiation over carbon material structure is frequently the subject of investigations due to a wide range of applications of these materials, in the aerospace industry for instance as radiation shield materials to protect personal and equipment from the damaging effect of radiation ${ }^{10}$. CNT yarns are lightweight, stiff, strong, ductile and electrically conductive materials that can be applied in wide range of multifunctional materials. Spacecrafts that enter into low or above orbit are subject to high radiation risk. It becomes difficult to develop new material to manufacture spacecrafts that are in frequent spaceflights and with long duration without consider the radiation damage in the material structure . The damage within the carbon structure may compromise the mechanical properties as well as electric and thermal conduction. The integrity CNT yarn structure plays a very important role in most applications. For example, Abot et al., 2014 propose an alternative method of strain monitoring and damage detection in composite material using CNT yarn. In this experiment, CNT yarn was used as a sensor integrated distributed in a laminate composite material to forming a continuous sensor circuit taking advantage of piezoresistive response that CNT yarn exhibit. The integrity of this yarn is very important to perform this measurement ${ }^{11}$.

In this paper, we studied the effect of electon beam and gamma irradiation on the mechanical properties and morphology of CNT yarns. The samples were characterized by Raman spectroscopy, scanning electron microscopy (SEM) and tensile tests to evaluate the effect of radiation on carbon structure, morphology and mechanical property, respectively.

\section{Materials and Methods}

CNT fiber was produced through a proprietary process by the University of Cincinnati. The fibers mounted on cardboard and a small tension was placed on the fibers ends so that the fiber kept straight during the radiation process. The samples were protected with aluminum foil during the radiation process.

One part of the CNT yarn sample was irradiated with a direct accelerator operated by the Instituto de Pesquisas Energeticas e Nucleares (IPEN- Sao Paulo/Brazil). 3 samples of CNT yarn were irradiated in air at doses of 400, 600 and 800 kGys with an industrial electron accelerator Dynamitron, from Radiation Dynamics Inc., model DC 1500/25-JOB 188 operating with the following parameters: beam energy 0.546 $\mathrm{MeV}$, pulse current $3.2 \mathrm{~mA}, 5 \mathrm{kGy} /$ pass with a dose rate of
$22.37 \mathrm{kGy} / \mathrm{s}$. The doses 400, 600 and $800 \mathrm{kGy}$ corresponds to total time of the sample under the beam of approximately $17.6 \mathrm{~s}, 26.4 \mathrm{~s}$ and $35.2 \mathrm{~s}$, respectively.

The other part of the CNT yarn sample was also irradiated in air by gamma radiation process in a Cobalt- 60 multipurpose irradiator plant at a dose rate of $6 \mathrm{kGy} / \mathrm{h}$.

Raman analyses were carried out in a Horiba JobinYvon T64000 Raman spectrometer equipped with a peltier cooled CCD with excellent sensitivity from 200-1000 nm and using a $600 \mathrm{gr} / \mathrm{mm}$ grating. The samples were deposited onto a glass slide, and the spectra were collected using a 50x objective in a backscattering configuration. The excitation energy was $2.33 \mathrm{eV}$ from the $532.1 \mathrm{~nm}$ line of an Argon laser. For each sample, a set of 5 spectra were collected at different points in the interval of $300 \mathrm{~cm}^{-1}$ to $3000 \mathrm{~cm}-1$. All spectra were treated to subtract the background and the peaks were fitted using Lorentzian curves.

The tensile properties of CNT fibers were assessed on a MTS single filament tensile tester, with $25 \mathrm{~mm}$ gauge length and at a $0.2 \mathrm{~mm} / \mathrm{min}$ extension rate (ASTM C1557). Ten specimens from each yarn sample were tested, and the average of the test results is reported. The yarn diameter (D) was measured using 500x optical microscope and was used to calculate the yarn specific strength.

The morphology of the CNT fibers was investigated using a Zeiss Auriga dual beam focus ion beam and scanning electron microscope (SEM) in which electron and ion beam can be used simultaneously. The focused ion beam (FIB) is generated from gallium liquid metal ion source with resolution of $7 \mathrm{~nm}$ at $30 \mathrm{Kv}$ acceleration voltages. The electron beam is generated from FEG electron source with high resolution SEM $1 \mathrm{~nm}$ at $15 \mathrm{KV}$ and $1.9 \mathrm{~nm}$ at $1 \mathrm{Kv}$.

\section{Results}

In Figure 1 are presented the results of mechanical strength of the CNT yarn that was electron beam (e-beam) irradiated at 400, 600 and $800 \mathrm{kGy}$ and gamma irradiated at dose of $100 \mathrm{kGy}$. The irradiated samples with electron beam at doses of 400, 600 and $800 \mathrm{kGy}$ had a decrease in the strength from $219.60 \pm 18.90 \mathrm{MPa}$ for pristine yarn to $108.86 \pm 23.77,153.15 \pm 21.63,170.50 \pm 25.78 \mathrm{MPa}$, respectively. The sample irradiated with gamma in air at dose of $100 \mathrm{kGy}$ had the strength increased slightly as compared with the pristine sample and an increase in the elasticity modulus from $8.79 \pm 1.19$ to $19.63 \pm 2.02 \mathrm{GPa}$ as compared to CNT pristine yarn. Elastic modulus is less sensitive to radiation because there are $\mathrm{C}$ double bonds around the defects on graphite structure which is very easily broken by the radiation process and produce free radicals. This new free radical combines randomly to reduce the amount of defects in carbon structure and this change the orientation of some carbon nanotube. Due to prolonged irradiation, the inter-wall distance decrease and larger crystallites are 
formed due to chemical bond formed between individual CNTs, leading to less flexibility compared with pristine yarn $^{12}$. On the other hand, the probability of cross-link can be reduced for the samples irradiated in air with high energy e-beam due oxidation and creation of larger defects in plane that can drastically affect the strength that is very sensitive to clustering of vacancies ${ }^{9}$. The oxidation within the structure and the deposition of amorphous carbon leads to stress concentration.

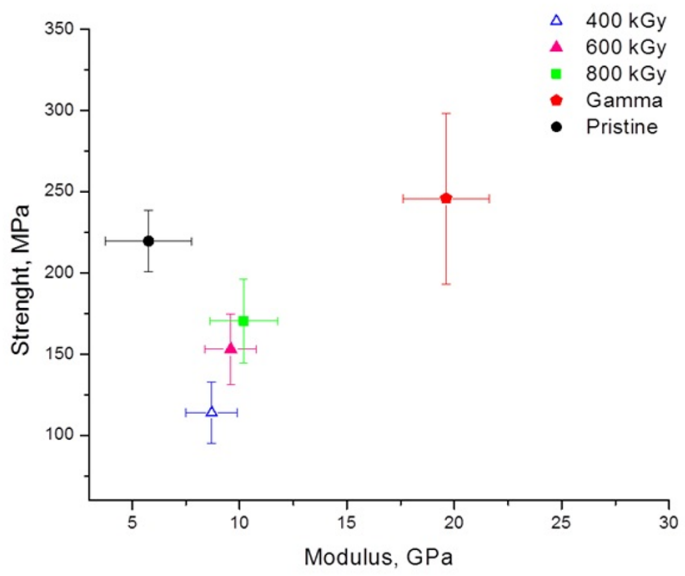

Figure 1. Tensile strength versus modulus of the yarn pristine and 400, 600, $800 \mathrm{kGy}$ e-beam-irradiated and $100 \mathrm{kGy}$ gamma irradiated CNTY samples.

Hiremath et al. studied the effect of e-beam irradiation at lower doses on CNT yarn. The samples were irradiated with $3.8 \mathrm{MeV}$ beam energy, with dose rate of $5 \mathrm{kGy} / \mathrm{sec}$ at dose of $100,200,300$ and $400 \mathrm{kGy}$. The tensile test results for these samples showed that the damage and scission process were predominant and reduced the strength drastically compared to pristine yarn. The FIB image of CNT yarn irradiated at $100 \mathrm{kGy}$ with $3.8 \mathrm{MeV}$ beam energy is presented in Figure 2. This sample had the strength reduced to $93.07 \pm$ $18.9 \mathrm{MPa}$ and modulus reduced to $4.44 \pm 0.57$ compared to $219.60 \pm 18.9 \mathrm{MPa}$ for strength and $5.77 \pm 2.00 \mathrm{GPa}$ for modulus of pristine CNT yarn. It is clear the empty spaces and some disorganization within the yarn structure hinders the load transfer. The CNT yarn irradiated at $300 \mathrm{kGy}$ had a slight improvement on the elastic modulus due to crystallite formation $^{13}$.

The SEM images presented in Figure 3 show the morphology of the yarn irradiated at $600 \mathrm{kGy}$ with electron beam. It can be noticed carbonaceous debris on the sample surface generated from the oxidation and, also disorganization in the alignment of the fiber leading to empty spaces within the yarn structure (Figure $3 a$ and $b)^{13}$. The oxidation could take place because the reaction with heteroatom from the environment. All these defects are points of stress concentration that contribute for such low strength ${ }^{13}$.

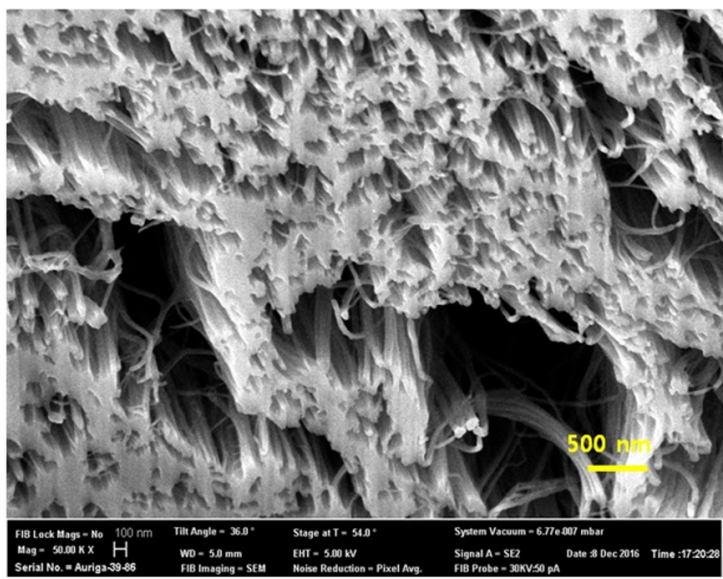

Figure 2. FIB images of the CNT yarn irradiated with $3.8 \mathrm{MeV}$ beam energy, with dose rate of $5 \mathrm{kGy} / \mathrm{sec}$ at dose of $100 \mathrm{kGy}{ }^{13}$.

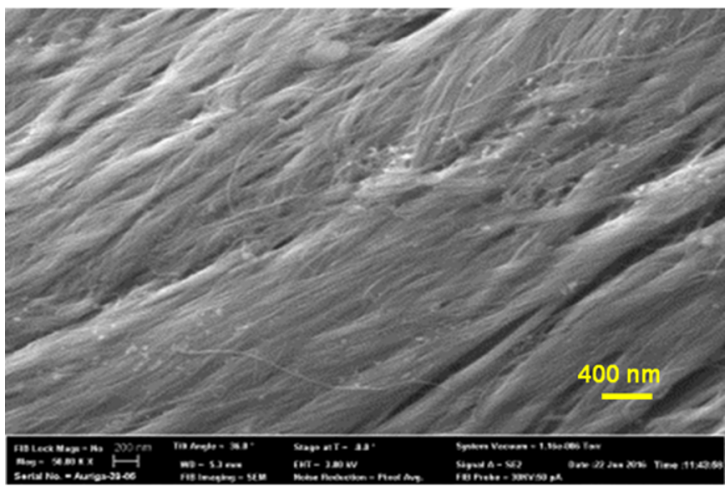

Figure 3. SEM images of CNT yarn e-beam irradiated at 600 $\mathrm{kGy}-\mathrm{a}$ and $\mathrm{b}$.

In Figure 4 SEM images are presented from the pristine samples (Figure 4a) and the samples e-beam irradiated at $800 \mathrm{kGy}$ (Figure 4b, c and d). The yarn irradiated at 800 kGy had a slight improvement on strength and modulus compared with the yarn irradiated at $400 \mathrm{kGy}$ (Figure1). Under a high dose radiation process a sample of CNT yarn can go through different defects created within yarn structure. These defects, such as yarn split, loose of alignment as well as cross linking. Some cross linking could be responsible for this slight improvement of the strength when the sample were irradiated at $800 \mathrm{kGy}$. The irradiation can push one carbon atom out of the graphene plane and crosslink the neighbors' graphene layers enhancing the load transfer ${ }^{7,9}$. This is consequence of the recombination process promoted by Wigner energy released from the nearest neighbor Frankel pair of defects or interstitial defect formed when the target atom was dislocated (Figure 4b). On the other hand, part of the sample shows yarn bundle split and loose of alignment that are responsible for a decrease on mechanical properties (Figure 4c). It is possible to note in Figure 4d some interconnection (welding process) among MWNT on the surface. The e-beam heating, due high energy concentration on a small volume of the sample, should be considered, however the 

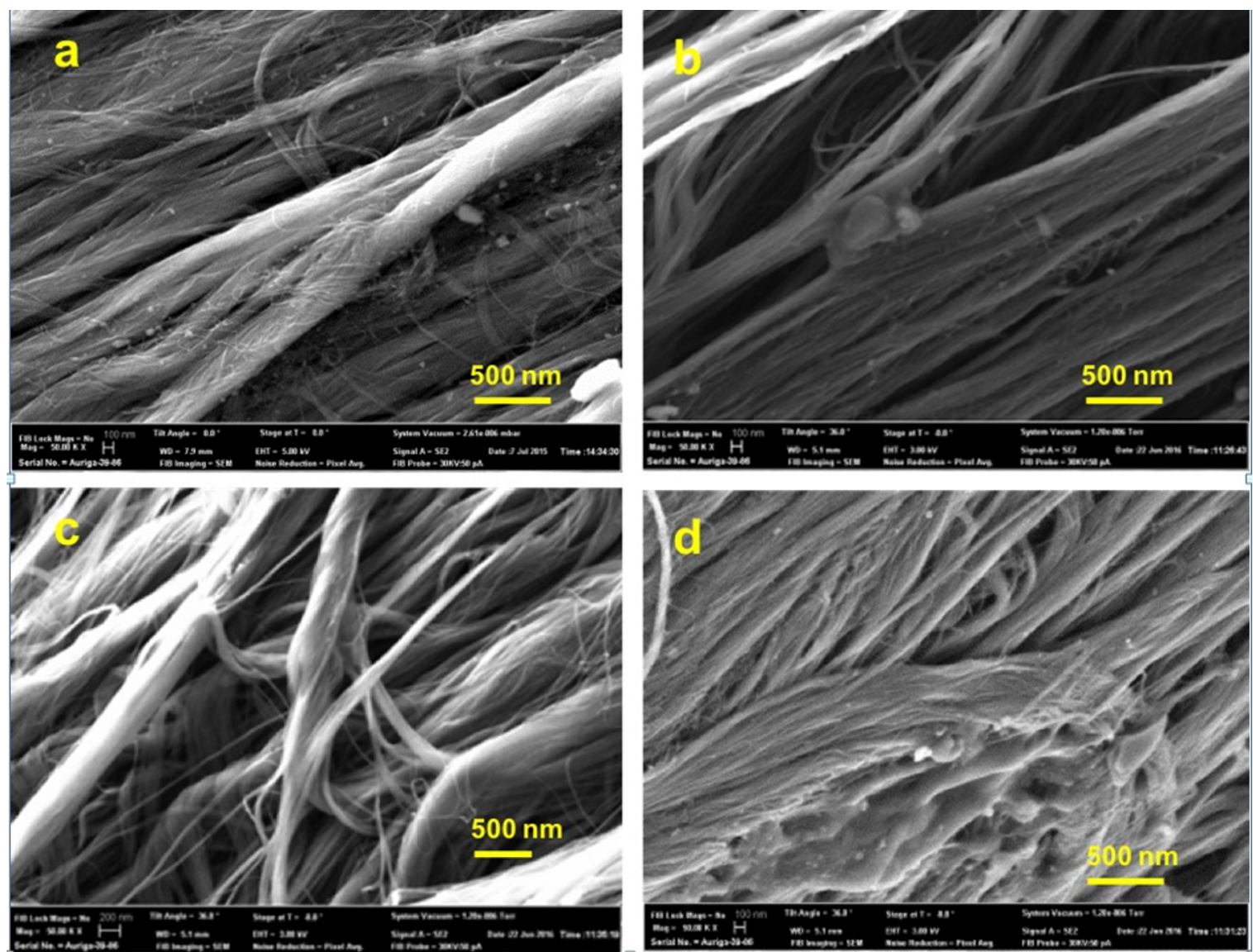

Figure 4. SEM images of CNT yarn e-beam irradiated at $800 \mathrm{kGy}$.

high temperature will not create junction among CNTs. The irradiation process induces vacancies and energy gain by dangling bond saturation and this makes possible to weld the tubes together ${ }^{14}$.

In Figure 5 is presented the SEM image of the CNT yarn irradiated with gamma ray. It is clear that the CNT yarn irradiated via gamma ray leads to smoother surface that probably because of higher proportion of cross linking that took place between adjacent CNT shells and tubes that resulted in better mechanical properties.

The CNT yarn irradiated with gamma ray at $100 \mathrm{kGy}$ shows better tensile strength and a significant improvement in the elastic modulus compared to the results of e-beam irradiated and pristine samples. The sample irradiated with gamma in air at dose of $100 \mathrm{kGy}$ had the strength increased slightly (245.52 \pm 52.55$)$ as compared with the pristine sample and an increase in the elasticity modulus from $8.79 \pm 1.19$ to $19.63 \pm 2.02 \mathrm{GPa}$ as compared to CNT pristine yarn. In addition, the yarn that was gamma irradiated showed a significant decrease in diameter (Table 1).

Safibonabi et al. reported the same result when MWCNTs (powder) were irradiated with gamma radiation in air at $100 \mathrm{kGy}$. The decrease in diameter and improvement of graphitization were reached but the samples irradiated at

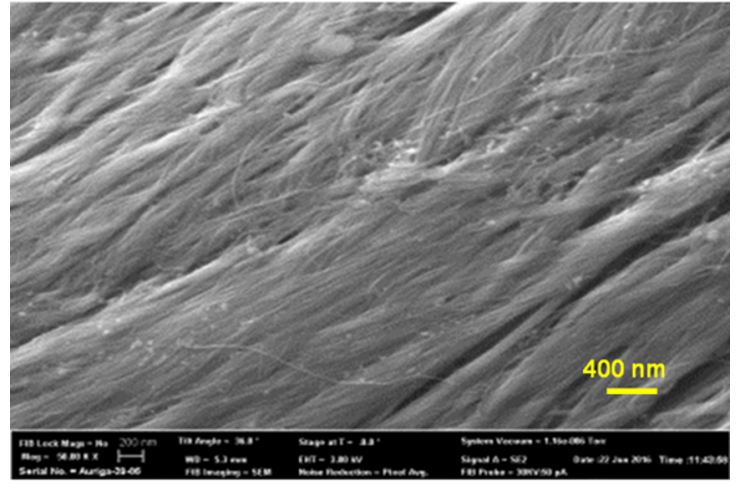

Figure 5. SEM images of CNT yarn gamma irradiated at $100 \mathrm{kGy}$.

Table 1. Diameter of CNTY irradiated samples

\begin{tabular}{lc}
\hline Samples irradiated (kGy) & Diameter (nm) \\
\hline 400 (electron beam) & $47.02 \pm 3.68$ \\
800 (electron beam) & $45.73 \pm 1.49$ \\
gamma & $36.98 \pm 1.84$ \\
\hline
\end{tabular}

$150 \mathrm{kGy}$ had the nanostructure of carbon destroyed ${ }^{15}$. Xu et al. also reported the same result for sample of MWCNTs was gamma irradiated in air $^{16}$. The penetrating power of gamma rays leads to significant rearrangement among carbon 

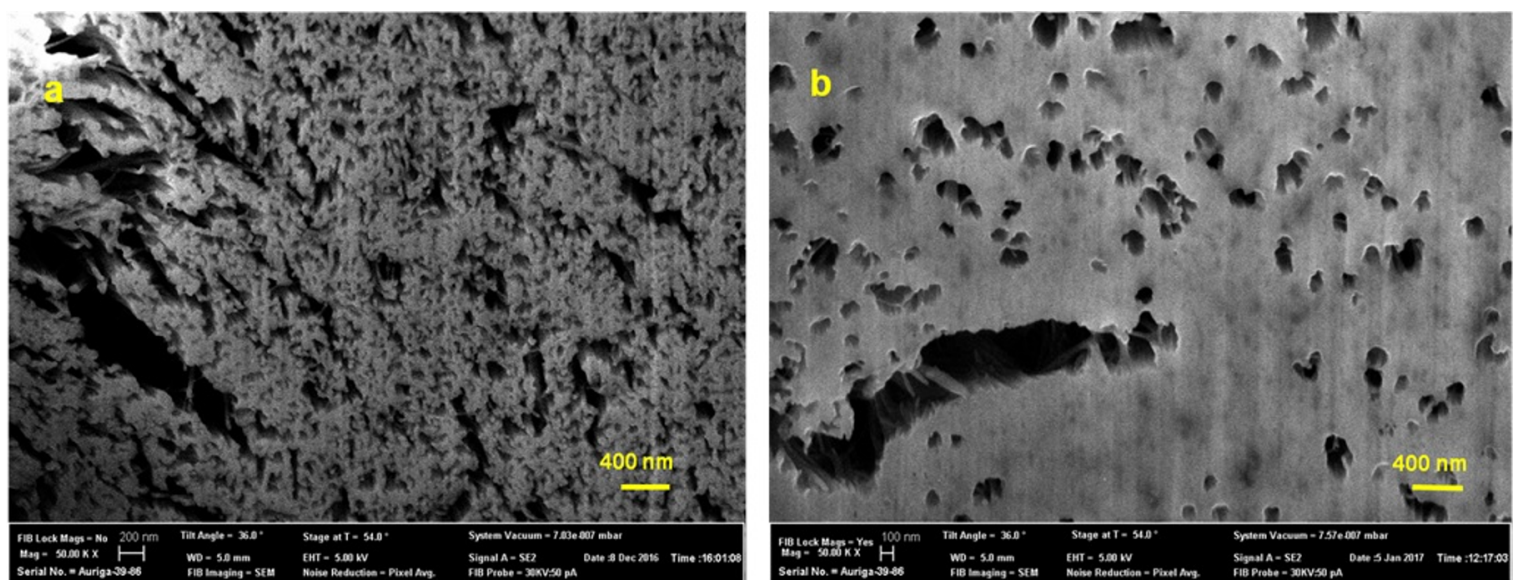

Figure 6. SEM images of a CNT yarn irradiated with ebeam at $800 \mathrm{kGy}$ (a) and CNT yarn gamma irradiated with at $100 \mathrm{kGy}-\mathrm{cut}$ using a focused ion beam (FIB).

atoms and, as a consequence, leads to a reduction in defect concentration and/or formation of crosslinking between adjacent carbons. As a result, the interwall spacing decreases with better organized structure and less empty spaces. The formation of bridges between carbons via covalent bond may explain the cross linking process and the improvement in mechanical property due to the enhancement of the load transfer. Photoeletric effect, Compton scattering and pair production are three different ways that gamma rays interacts with matter but Compton scattering is the most expected effect for interaction of gamma rays with carbon based material ${ }^{17}$. The electrons ejected from Compton scattering effect are responsible not only for ionization process ${ }^{18,19}$, but also are responsible for carbon atom displacement. Those electrons have enough energy to create mobile interstitial carbon atoms in the confined space between neighbors' tubes and the disappearance of these interstitial atoms by recombination that leads to cross linking process. This cross linking is very well illustrated in FIB images presented in Figure $6 \mathrm{a}$ and $\mathrm{b}$. Unlike the CNT yarn irradiated with e-beam at $800 \mathrm{kGy}$ (Figure 6a), the FIB image of CNT yarn irradiated with gamma ray at $100 \mathrm{kGy}$ (Figure 6b) presented less empty spaces and better interaction between adjacent CNTs due cross linking process. The modifications promoted by gamma source happen not only on the surface of the yarn (Figure 5), but also inside of the yarn structure.

Raman spectroscopy results for the pristine CNT fiber and samples irradiated are shown in Figure 7 and Figure 8. The G, D and D' band corresponds to the $\mathrm{C}=\mathrm{C}$ in-plane stretching mode, lattice of defects and finite crystal size (nanocarbon), respectively.The Raman spectra were normalized on the basis of G-band intensity. The D (aprox. $1350 \mathrm{~cm}^{-1}$ ), G (aprox. $1580 \mathrm{~cm}^{-1}$ ) and D' (aprox. $1600 \mathrm{~cm}^{-}$ $\left.{ }^{1}\right)$ band positions are presented in the graph. The intensity ratio of $\mathrm{D}$ band to $\mathrm{G}$ band (ID/IG) in the Raman spectra has been widely used to evaluate the disorder in $\mathrm{sp}^{2}$ hybridized carbon systems ${ }^{20}$. The fitting of all spectra was made with

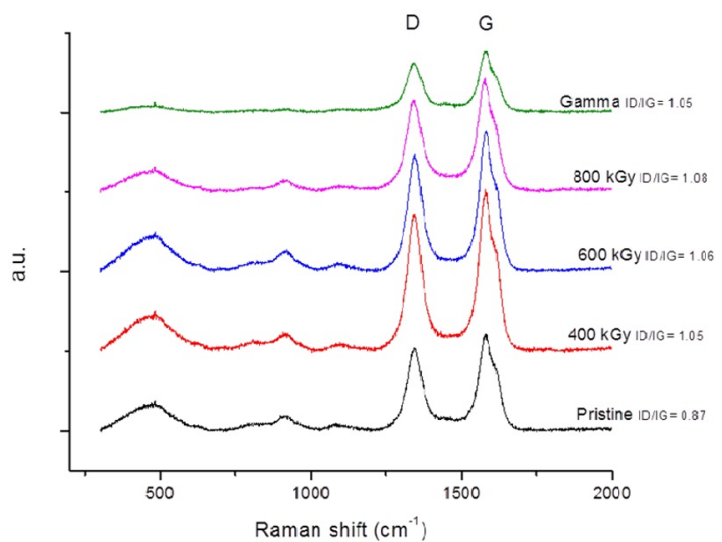

Figure 7. Raman spectroscopy results for CNTY pristine and CNTY e-beam irradiated at 400,600,800 kGy and gamma irradiated at $100 \mathrm{kGy}$.

Lorentzian peaks and the ID/IG ratio was calculated by the area of the curves for each sample and ID/IG increases with an increase in the number of defects on the sidewall. Radiation process introduces point of defects by creation of $\mathrm{sp}^{3}$ defects and the disruption of $\pi-\pi$ conjugation of the graphitic structure. There is an increase in ID/IG ratio from 0.87 for pristine CNT yarn up to 1.08 indicating increase in defects in the samples irradiated by both radiation sources. The D' band area increased from 11.75 a.u. for the pristine sample to 23.84 a.u. for the sample irradiated to $800 \mathrm{kGy}$ due to larger crystallites formation which leads to higher elastic modulus compared with pristine. The CNT yarn irradiated with gamma source had D' band area decreased to 6.07 a.u. indicating the reduction of carbon nanoclusters. We can observe that FWHM of the G peak decreased from 52.26 $\mathrm{cm}^{-1}$ to $49.04 \mathrm{~cm}^{-1}$ for pristine and gamma irradiated yarn, respectively what may demonstrate the increasing quality ${ }^{21}$. The vacancies introduced from the displacement of carbon atoms due to irradiation- induced- sputtering can be stabilized by creating pentagon-heptagons defects. Because the ability 

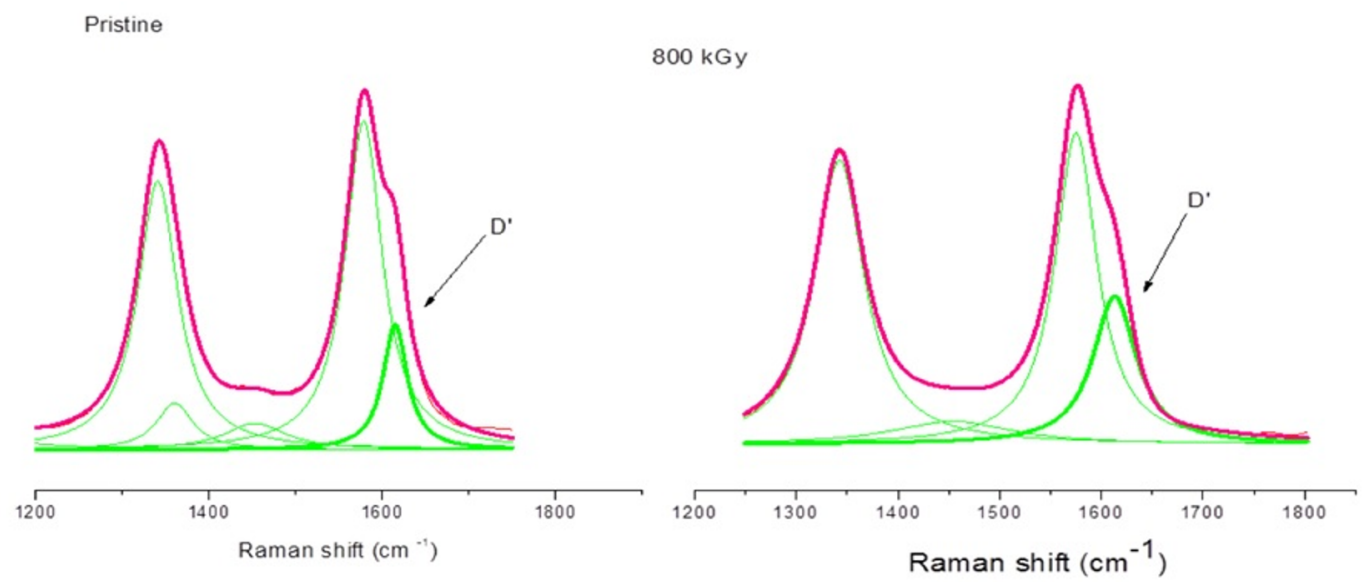

Gamma

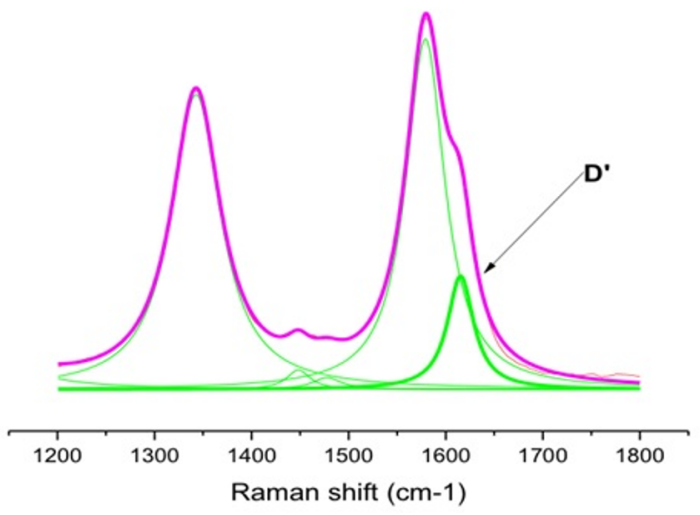

Figure 8. Raman spectra of CNTY from 1200 to $1800 \mathrm{~cm}^{-1}-\mathrm{D}, \mathrm{G}$ and D'peaks.

of CNTs to self repair, the carbon atoms displaced from the graphene plane stabilize through crosslinking process between neighboring graphene layers ${ }^{6,19,22}$.

\section{Conclusion}

CNT yarn was electron beam (e-beam) irradiated at 400, 600 and $800 \mathrm{kGy}$ and gamma irradiated at dose of $100 \mathrm{kGy}$. It was observed that the strength of the samples irradiated with e-beam decrease drastically compared with pristine sample. However, there is an improvement of elastic modulus for the samples irradiated with gamma ray and slight improvement for the samples irradiated with e-beam. The quality of the CNT yarns that was gamma irradiated with absorbed dose of $100 \mathrm{kGy}$ remains the same with improvement of $123 \%$ of the Young's modulus. The penetrating power of gamma rays leads to significant rearrangement among carbon atoms and, consequently, leads to reduce the defect concentration and/or formation of crosslinking between adjacent carbons. As a result, the interwall spacing decreases with better organized structure and less empty spaces with decrease of the diameter. The CNT yarn irradiated with gamma rays had
D' band area decreased to 6.07 a.u. indicating the reduction of carbon nanoclusters. The formation of bridges between carbons displaced from the graphene plane via covalent bond may explain the cross linking process between neighboring graphene layers, enhancing the load transfer, consequently the improvement of mechanical property. The drastic decrease in strength of the CNT yarn irradiated with e-beam can be addressed accumulation of vacancies, reactions with heteroatom from the environment (oxidation) that leads to stress concentration and larger crystallites formation that may slight improve the elastic modulus but not the strength.

\section{Acknowledgment}

We would like to thank Conselho Nacional de Desenvolvimento Científico e Tecnológico (CNPq) for a postdoctorate scholarship and NASA for the financial support provided (NASA EPSCoR Cooperative Agreement NNX13AD41A). Also we would like to thanks Institute for Nuclear and Energy Research for the radiation and Material Science and Technology Division at Oak Ridge National Laboratory for the mechanical tests. 


\section{References}

1. Wu AS, Chou TW. Carbon nanotube fibers for advanced composites. Materialstoday. 2012;15(7-8):302-310.

2. Behabtu N, Green MJ, Pasquali M. Carbon nanotube-based neat fibers. Nanotoday. 2008;3(5-6):24-34.

3. Min J, Cai JY, Sridhar M, Easton CD, Gengenbach TR, McDonnell J, et al. High performance carbon nanotube spun yarns from a crosslinked network. Carbon. 2013;52:520-527.

4. Coleman JN, Klan U, Blau WJ, Gun'ko YK. Small but strong: A review of the mechanical properties of carbon nanotubepolymer composites. Carbon. 2006;44(9):1624-1652.

5. Yu Q, Alvarez NT, Miller P, Malik R, Haase MR, Schulz M, et al. Mechanical Strength Improvements of Carbon Nanotube Threads through Epoxy Cross-Linking. Materials (Basel). 2016;9(2). pii:E68.

6. Hao X, Yonggen L, Mouhua W, Xianying Q, Weizhe Z, Jian L. Effect of gamma-irradiation on the mechanical properties of polyacrylonitrile-based carbon fiber. Carbon. 2013;52:427-239.

7. Kis A, Csányi G, Salvetat JP, Lee TN, Couteau E, Kulik AJ, et al. Reinforcement of single-walled carbon nanotube bundles by intertube bridging. Nature Materials. 2004;3:153-157.

8. Solá F. Electrical properties of pristine and electron irradiated carbon nanotube yarns at small length scales. Modern Chemistry \& Applications. 2014;2(1):116.

9. Filleter T, Espinosa HD. Multi-scale mechanical improvement produced in carbon nanotube fibers by irradiation cross-linking. Carbon. 2013;56:1-11.

10. Mylvaganam K, Zhang LC. Fabrication and application of polymer composites comprising carbon nanotubes. Recent Patents on Nanotechnology. 2007;1(1):59-65.

11. Abot JL, Winter K, Mortin SP, Borges de Quadros H, Le HH, Renner DC, et al. Localized detection of damage in laminated composite materials using carbon nanotube yarn sensors. Journal of Multifunctional Composites. 2014;2(4):217-226.
12. Park OK, Choi H, Jeong H, Jung Y, Yu J, Lee JK, et al. Highmodulus and strength carbon nanotube fibers using molecular cross-linking. Carbon. 2017;118:413-421.

13. Hiremath N, Lu X, Evora MC, Naskar A, Mays J, Bhat G. Effect of solvent/polymer infiltration and irradiation on microstructure and tensile properties of carbon nanotube yarns. Journal of Materials Science. 2016;51(22):10215-10228.

14. Krasheninnikov AV, Banhat F. Engineering of nanostructured carbon materials with electron or ion beams. Nature Materials. 2007;6:723-733.

15. Safibonab B, Reyhani A, Golikand AN, Mortazavi SZ, Mirershadi $\mathrm{S}$, Ghoranneviss M. Improving the surface properties of mutiwalled carbon nanotubes after irradiation with gamma rays. Applied Surface Science. 2011;258(2):766-773.

16. Xu Z, Chen L, Liu L, Wu X, Chen L. Structural changes in multi-walled carbon nanotubes caused by $\gamma$-ray irradiation. Carbon. 2011;49(1):339-351.

17. Xu Z, Huang Y, Min C, Chen L, Chen L. Effect of $\gamma$-ray radiation on the polyacrylonitrile based carbon fibers. Radiation Physics and Chemistry. 2010;79(8):839-843.

18. Xu Z, Liu L, Huang Y, Sun Y, Wu X, Li J. Graphitization of polyacrylonitrile carbon fibers and graphite irradiated by $\gamma$ rays. Materials Letters. 2009;63(21):1814-1816.

19. Li B, Feng Y, Ding K, Qian G, Zhang X, Zhang J. The effect of gamma ray irradiation on the structure of graphite and multiwalled carbon nanotubes. Carbon. 2013;60:186-192.

20. Dresselhaus MS, Jorio A, Souza Filho AG, Saito R. Defect characterization in graphene and carbon nanotubes using Raman spectroscopy. Philosophical Transactions of the Royal Society A: Mathematical, Physical and Engineering Sciences. 2010;368(1932):5355-5377.

21. Eigler S, Dotzer C, Hirsch A. Visualization of defect densities in reduced grapheme oxide. Carbon. 2012;50(1):3666-3673.

22. Hulman M, Skákalová V, Roth S, Kuzmany H. Raman spectroscopy of single-wall carbon nanotubes and graphite irradiated by $\gamma$ rays. Journal of Applied Physics. 2005;98(2):024311. 